\title{
Characterizing Subjective Noisiness in Hospital Lobbies
}

\author{
Chiung-Yao CHEN \\ Department of Architecture, Chaoyang University of Technology \\ 168, Gifeng E. Rd, Wufeng Dist., Taichung Taiwan; e-mail: chychen@cyut.edu.tw
}

(received January 1, 2015; accepted March 23, 2015)

\begin{abstract}
The present study was conducted in the lobbies of 16 Taiwanese urban hospitals to establish what contributes to the degree of noisiness experienced by patients and those accompanying them. Noise level measurements were then conducted by $15 \mathrm{~min}$ equivalent sound pressure levels $\left(L_{\text {Aeq, } 15 \mathrm{~m}, \mathrm{~dB}}\right)$ during daytime hours. The average $L_{A e q}$ itself was found to be poorly related to perceived noisiness. Levels variations were better correlated, more continual noise may actually be perceived as noisier. According to the findings of a multiple linear stepwise regression model $\left(r=0.91, R^{2}=0.83\right)$, the 3 independent variables shown to have the largest effects on perceived noisiness were 1) $1 /\left(L_{5}-L_{95}\right), 2$ ) effective duration of the normalized autocorrelation function $\left(\tau_{e}, h\right)$, of all $L_{A e q, 15 \mathrm{~m}}$ over $9-17$, and 3$)$ percentile loudness, $N_{5,15 \mathrm{~m}}$. These results resemble previous studies that had assumed that a larger fluctuation of noise level corresponds to less annoyance experienced for mixed traffic noise studied in a laboratory situation. As an advanced approach, for hospital noise that consisted of 12 audible noise events, subjective noisiness were evaluated by the noise time structure analyzed by autocorrelation with loudness and levels variation.
\end{abstract}

Keywords: noisiness, hospital, annoyance, loudness, autocorrelation function, reverberation time.

\section{Introduction}

\subsection{Psychologically annoyed by noise}

The noise in hospitals is detrimental to the recovery of patients, who need an environment quieter than they usually experience in their daily lives. The noise in hospitals also causes distractions to the hospital staff, increasing the risk of medical accidents. Nevertheless, verbal communications in a hospital lobby constitute a necessary behavior at registration desk, dispensary counters, and outpatient service counters. Those actual procedures that patients undergo normally lasting longer than 20-30 min in the hospital lobbies can be an uncomfortable experience in Taiwan. While it has previously been reported that the conversational din represented by the noise in hospital lobbies is an underlying cause of stress among patients, staff, and visitors, the amount of research conducted on noise in hospital lobbies remains limited (TOPF, 1988; RYHERD et al., 2008). This paper focuses on noise-related variables in heavily trafficked hospital lobbies including noise levels at registration desk, at dispensary counters, and in emergency departments. The particular noise sources in hospital lobbies consist primarily of people, machinery, and medical appliances; however the specific acoustical characteristics of hospital lobbies have not been well established.

Bush-VishniaC et al. (2005) reviewed the extensive literature on hospital noise levels from the last 4050 years. Their review revealed that measurements of sound pressure levels in hospitals have significantly exceeded the maximum noise levels allowed under World Health Organization (WHO) guidelines (BERGLUND et al., 1999, page 15). Specifically, the WHO guidelines recommend that noise levels in hospital and ward rooms be kept under $30 L_{A e q}$ in order to allow verbal communications to be intelligible, and kept below $40 L_{A \max }$ to avoid the critical health effect of sleep disturbance. Most of the data from previous research has shown noise levels $20-40 \mathrm{~dB}$ higher than the maximums recommended by the WHO guidelines (RYHERD et al., 2008). The daily activities in hospital have resulted in excessive noise generated from sources such as enormous air-conditioners, medical treatments involving noisy portable carts, conversations, and even TV sounds in lobbies. Recently, many hospitals in Taiwan have opened a convenience store in their lobbies, thus greatly increasing the complexity of the noise profile in these places (Table 1). Furthermore, hospitals run 
Table 1. The sizes of the lobbies and the nature of adjacent spaces in the hospitals surveyed.

\begin{tabular}{|c|c|c|c|c|c|c|c|c|c|c|c|c|c|}
\hline & NP & $\mathrm{AR}\left[\mathrm{m}^{2}\right]$ & $\mathrm{VO}\left[\mathrm{m}^{3}\right]$ & $\mathrm{CH}[\mathrm{m}]$ & NS & $\mathrm{NE}$ & NB & OD & ED & $\mathrm{EV}$ or $\mathrm{ES}$ & $\mathrm{CD}$ & MRI & $\mathrm{CT}$ \\
\hline $\mathrm{A}$ & 42 & 100 & 280 & $2.8 \#$ & 20 & 3 & $401(2)$ & $\mathrm{y}$ & & & & $1 \mathrm{~T}$ & 64 \\
\hline $\mathrm{B}$ & 35 & 295 & 1033 & $3.5 \#$ & 80 & 3 & $396(7)^{*}$ & & & $\bar{y}$ & & $1.5 \mathrm{~T}$ & 64 \\
\hline $\mathrm{C}$ & 28 & 357 & 964 & $2.7 \#$ & 70 & 3 & $444(5)^{*}$ & $\mathrm{y}$ & $\mathrm{y}$ & $\mathrm{y}$ & & $1 \mathrm{~T}$ & 64 \\
\hline $\mathrm{D}$ & 44 & 300 & 900 & 3.0 & 60 & 2 & $243(3)$ & & & $\mathrm{y}$ & & & 64 \\
\hline $\mathrm{E}$ & 39 & 300 & 900 & 3.0 & 140 & 3 & $237(3)^{*}$ & $\mathrm{y}$ & $\mathrm{y}$ & $\mathrm{y}$ & & & \\
\hline $\mathrm{F}$ & 49 & 168 & 588 & $3.5 \#$ & 50 & 5 & $638(2)$ & & & & $\mathrm{y}$ & $1 \mathrm{~T}$ & 64 \\
\hline $\mathrm{G}$ & 29 & 197 & 887 & $4.5 \#$ & 70 & 5 & $763(17)^{*}$ & $\mathrm{y}$ & $\mathrm{y}$ & $\mathrm{y}$ & & $1 \mathrm{~T}$ & 64 \\
\hline $\mathrm{H}$ & 31 & 287 & 718 & $2.5 \#$ & 120 & 2 & $585(3)$ & $\mathrm{y}$ & $\mathrm{y}$ & $\mathrm{y}$ & & $1.5 \mathrm{~T}$ & 64 \\
\hline $\mathrm{I}$ & 22 & 410 & 12340 & $2.8 \#$ & 150 & 3 & $517(7)^{*}$ & $\mathrm{y}$ & $\mathrm{y}$ & $\mathrm{y}$ & $\mathrm{y}$ & $1.5 \mathrm{~T}$ & 64 \\
\hline $\mathrm{J}$ & 32 & 1300 & 12900 & $3.0 \#$ & 150 & 2 & $373(4)^{*}$ & $\mathrm{y}$ & $\mathrm{y}$ & $\mathrm{y}$ & $\mathrm{y}$ & $1.5 \mathrm{~T}$ & 64 \\
\hline $\mathrm{K}$ & 28 & 713 & 3919 & $5.5 \#$ & 108 & 3 & $116(10)^{*}$ & $\mathrm{y}$ & $\mathrm{y}$ & $\mathrm{y}$ & $\mathrm{y}$ & $1 \mathrm{~T}$ & 64 \\
\hline $\mathrm{L}$ & 44 & 197 & 788 & $4.0 \#$ & 60 & 2 & $235(9)^{*}$ & $\mathrm{y}$ & $\mathrm{y}$ & $\mathrm{y}$ & $\mathrm{y}$ & & 64 \\
\hline $\mathrm{M}$ & 36 & 310 & 930 & $3.0 \#$ & 110 & 5 & $839(3)$ & $\mathrm{y}$ & & & & $1.5 \mathrm{~T}$ & 64 \\
\hline $\mathrm{N}$ & 41 & 300 & 2250 & $7.5 \#$ & 72 & 3 & $560(10)^{*}$ & $\mathrm{y}$ & $\mathrm{y}$ & $\mathrm{y}$ & & $1.5 \mathrm{~T}$ & 64 \\
\hline $\mathrm{O}$ & 40 & 276 & 828 & 3.0 & 75 & 5 & $258(7)^{*}$ & & & $\mathrm{y}$ & $\mathrm{y}$ & & 64 \\
\hline $\mathrm{P}$ & 52 & 240 & 843 & $7.5 \#$ & 60 & 5 & $717(4)$ & $\mathrm{y}$ & $\mathrm{y}$ & $\mathrm{y}$ & $\mathrm{y}$ & $1.5 \mathrm{~T}$ & 64 \\
\hline
\end{tabular}

NP - Numbers of participants for subjective noisiness survey;

AR - Area of lobby hall but hallway, stores, elevator facilities, etc. were excluded;

VO - Volume of lobby hall but hallway, stores, elevator facilities, etc. were excluded;

$\mathrm{CH}$ - Ceiling height of lobby hall;

NS - Numbers of seats in the lobby;

NE - Number of entrances;

NB - Number of inpatients' bed and the amount of flooring of the lobby building in round bracket;

$\mathrm{EV}$ - Elevator;

ES - Escalator;

OD - Connected to outpatient department;

ED - Connected to emergency department;

CD - Connected to drug or convenience store;

\# - Hall ceiling was decorated by some absorptive treatments;

* - Wards were located at higher floors above the lobby in the same building;

MRI - Quantity of the magnetic field (Tesla), magnetic resonance imaging;

CT - Slices of X-ray image, computerized tomography.

huge mechanical infrastructures, such as the magnetic resonance imaging (MRI) and computerized tomography (CT) medical facilities listed in Table 1. For convenience, to dampen their sounds and vibrations, and for the purposes of emergencies, these pieces of equipment have routinely been sited on the first floor (1F) or basement floor (BF). Such facilities, however, drastically augment noise over a frequency range of 500-2000 Hz measured in operating rooms and with spectral peaks of $250 \mathrm{~Hz}$ (MCJuRY, 1995). However, in our previous survey, the noise levels of these magnet facilities were under $49 L_{A e q}$ at 1-min intervals measured at the hallway right outside of these examination rooms.

The effects of noise on patients and staff is a major concern in hospital lobbies, but, most previous studies have focused only on the noise levels of individual hospital units, or have detailed the acoustical properties of medical equipment. Only a few studies, in fact, have provided any information about annoyance and noise perceptions in terms of road-traffic composition (SANDROCK et al., 2010; ABO-QUDAIS, ABU-
QDAIS, 2005). Those studies concluded that annoyance is highly dependent on the actual noise sources in complex traffic scenarios but provided only limited evaluations regarding the complicated hospital noise disturbances in the lobbies studied.

\subsection{The nature of the urban hospital lobbies}

In hospital lobbies, noises generated by medical activities may be combined with noises from adjacent hallways or escalators, all of which are required to clear the spatial guidance regulations for urban hospitals in Taiwan (Table 1). Unfortunately, however, these arrangements tend to create wave tunnels, with sounds travelling down the hallways and into lobbies and negatively affecting waiting patients and the people accompanying them. In addition, the floors and walls typically consist of reflective, solid materials designed for durability, cleanliness, and convenience. As indicated in Table 1, the ceilings of many lobbies contain absorbent panels that serve visual aesthetic and acous- 
tic purposes; however, even though hard surfaces are not essential in lobbies, other often used materials include fully reinforced concrete or rock surfaces. The result, as noted by Bush-VishniaC et al. (2005), is that hospitals are noisy and getting noisier at an alarming rate. WHO guidelines propose that reverberation times of over 1.0 s produce losses in speech discrimination and make speech perception more difficult, to the point of even causing listeners to strain (BERGLUND et al., 1999, page 6). This is an important issue in hospital lobbies because the vast majority of information is transmitted orally (for example, by nurses or other staff members speaking with patients and their families). In this study, we investigated the psychological effects of noise during daytime hours in 16 urban hospital lobbies with the aim of providing complicated noise evaluations based on a variety of acoustical descriptors used in previous works.

\subsection{Noise criteria}

\section{A. Statistical noise gauges}

Previous studies have employed equivalent sound pressure level $\left(L_{e q}\right)$ measurements to quantify noise levels according to the WHO guideline recommendations (Berglund et al., 1999, page 7). Some studies have focused on the variation of $L_{e q}$ over a 24-hour period (BUsh-Vishniac et al., 2005; YAMADA et al., 2003; Orellana et al., 2007; Kracht et al., 2007; Williams et al., 2007). For road-traffic noise GRIFFITHS, LANGDON (1968) proposed using a statistical level of $L_{N}$, with $L_{10}-L_{90}$ as the measure of variability. De Coensel et al. (2005) used $L_{5}-L_{95}$ as an effective descriptor of noise observations since they showed much more variation among statistical levels, while TORIJA et al. (2010) used a sound level variance which was calculated by the standard deviation of the sound levels. However, TORIJA et al. (2010) provided only limited psychological research regarding their models. These indicators provide an idea of the cumulative fluctuation levels from the average background noise. In other words, the psychological effects of noise were mainly brought about by the periodic or successive occurrence of noises. In addition, KaczMarek, Preis, (2010), investigated how the time-varying structure of road-traffic levels affects the noise-related annoyance judgments. They found that the highest annoyance ratings were obtained for even traffic distributions, and the most clustered distribution resulted in the lowest annoyance ratings. Additionally, different noise annoyance ratings for the same $L_{A e q}$ were caused by different mean percentile loudness in sone $\left(N_{10}\right.$ or $\left.N_{5}\right)$. They concluded that the average loudness, $N$, better correlates to annoyance ratings than the percentile value of loudness $N_{5}$, and that $N$ can be used as a noise index for time-varying noise. However, their four traffic noise structures were artificially created in a lab- oratory from single vehicle pass-by recordings. They do not seem capable of representing community noise, which is defined by WHO (BERGLund et al., 1999, page 55) as noise emitted from all sources, including road-traffic, industries, construction, public work, and neighborhoods; i.e. the real noises which occur in our environments. Whereas the findings reported by KacZmarek and Preis (2010) regard their experiences of the variance in road-traffic noise measures, we believed that statistical levels and percentile loudness of noise measurements would serve as an effective descriptor of the psychological noisiness for the complicated noise sources in hospital lobbies, too. Meanwhile, other measures such as the maximum and minimum noise levels, octave band levels, and, in particular, the sense of time-varying in noise levels continuity have been investigated by using autocorrelation function analysis (ANdo, Chen, 1996; SAto et al., 2007).

\section{B. Zwicker's loudness}

The Zwicker's loudness in hospital lobbies has also been investigated in previous studies (BERANEK, 1956; ISO 532, 1975), while TANG (1997), previously investigated objective and subjective evaluations of the air conditioning noises in offices. TANG (1997) found that the less commonly used Zwicker's loudness level performs better than the commonly adopted NC curves (BERANEK, 1989) when predicting the noise perceptions of office workers in air-conditioned landscaped offices. BERGLUnd et al. (2002) used Zwicker's loudness scale to investigate annoyance levels due to environmental noises. They found that Zwicker's specific loudness based on critical bands matched the listener's judgment of annoyances more accurately than $L_{A e q}$ levels. They presented 15 independent noise samples, such as "passing car", "office printer" etc., in pairs, each with a duration of $4 \mathrm{~s}$, using non-preference judgments. In referencing the abovementioned studies on loudness, we found that their noisiness research subjects were mostly subjected to specific individual noise sources. Similarly, the lobby noisiness in our study consisted of many real noises mixed together, rather than recordings of discrete separate noise sources.

\section{Autocorrelation function of noise levels}

On the other hand, the factors extracted from an autocorrelation function (ACF) usually signify a degree of similar repetitive features for a random signal (Ando, Chen, 1996; Sato et al., 2007). As shown in Fig. 1, the normalized ACF (NACF) curve indicates a sound similarity in the time domain, and the effective duration of the NACF, $\tau_{e}$ values $(h)$ indicates the slope of the NACF curve decay in the initial part (Ando, Chen, 1996). Sato et al. (2007) re- 


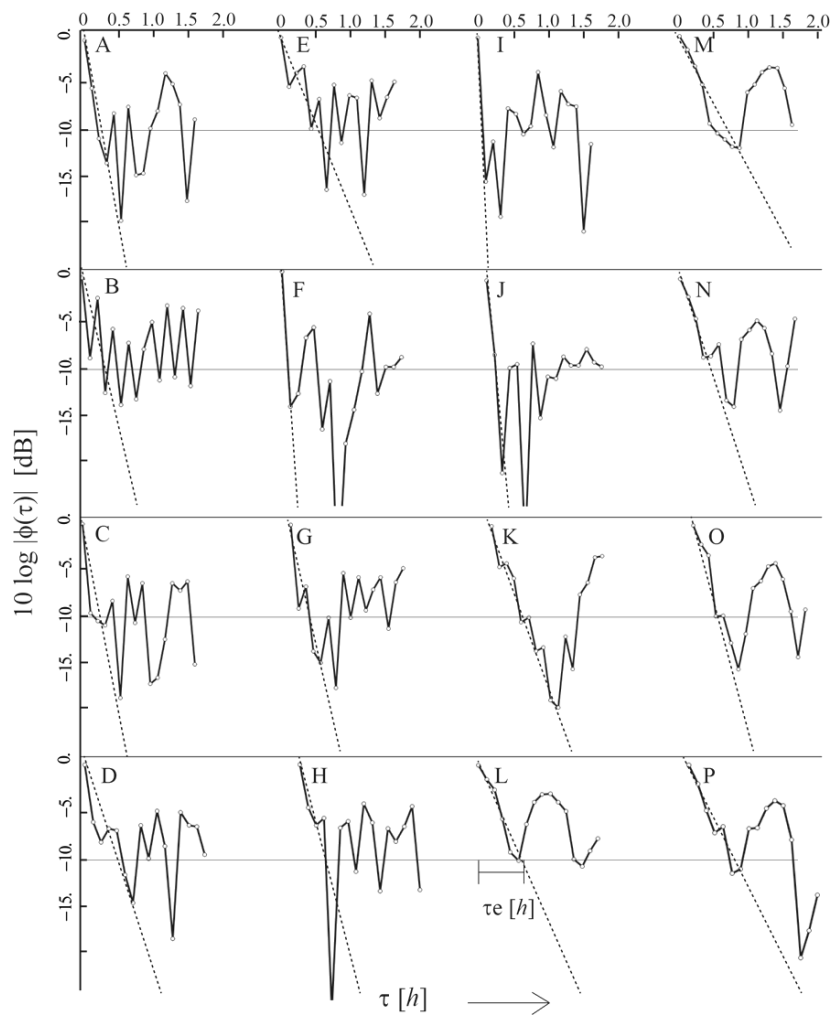

Fig. 1. A linear sum of noise levels of $L_{A e q}$ at 15-min intervals in the hospital lobby shows an initial decline in the envelope of the normalized autocorrelation function (NACF), and this decline can be fit to a straight line regression in a range of 0 to $-10 \mathrm{~dB}$ of the power of the NACF. The effective duration of NACF of noise levels $\left(\tau_{e}, h\right)$ is defined as it crosses to $-10 \mathrm{~dB}$ at that of delay.

ported that the ACF parameters of refrigerator noise energy and its fluctuations are important for evaluating noisiness. But their findings were limited in their subjective evaluation against the target noise. The emphasis of the present study is the interpretation of the NACF decay rate as an indicator of the degree of continuity of the noise levels in the lobbies. They did report that, according to their measurements, larger variations in decibels were perceived as less noisy.

\subsection{Psychological noisiness}

It is important to investigate subjective, as well as objective, responses to noise. YAMADA et al. (2003) performed post-occupancy evaluations of subjective responses to sound environments in hospital wards. Their analysis of a questionnaire survey showed that many inpatients pointed out that conversations and noisy medical equipment measured by $L_{A e q}$ at $15 \mathrm{~min}$ intervals were rated as unacceptable noises. Moreover, the responses of nurses showed a higher degree of annoyance than those of inpatients. Tsiou et al. (2008) focused on the opinions of medical personnel regard- ing noise in operating rooms where surgery preparations were made and which also served to isolate the surgical room from the corridor. The results of these subjective assessments of noise showed that the professional roles of individuals in the operating room influence their perceptions of noise. These results suggest that research involving the use of questionnaires for noise evaluation should avoid seeking answers from hospital personnel; consequently, responses regarding individual perceptions of noisiness in the present study were restricted to the visitors in the hospital lobbies.

To clarify the relationship between the objective measures and the subjective response to complex noise, a questionnaire survey and noise measurements were conducted in the lobbies of 16 urban hospitals in Taiwan. To measure the level of noisiness, visitors in the lobbies were asked to fill out questionnaires, since it would be difficult to evaluate such noisiness via the professional roles of the individuals working in hospitals (RYHERD, 2008; YAMADA et al., 2003; Tsiou et al., 2008).

\section{Methodology}

\subsection{Hospital survey}

16 urban hospitals located in various Taiwanese cities, all of which included service departments such as internal medicine, surgery, orthopedics, cardiology etc., were selected to participate in the survey. Each of these hospitals had outpatient services, an emergency department, examination rooms, operating rooms connected to their lobbies, and wards with more than 100 beds. However, hospital regulations in Taiwan require that inpatient wards should be located as far from the lobbies as possible; hence, those wards were located in different buildings to the extent possible according to the size of the overall hospital site. As indicated in Table 1 , however, most of the hospitals sited their ward departments in the same building above the lobbies. Meanwhile, hospitals hosting medical schools were excluded from this study, because their service departments, especially their emergency departments, were sited in separate buildings. Each of the hospitals surveyed is confined to a single site; large-sized hospitals consisting of multiple buildings were not included. The main entrance of each surveyed hospital is adjacent to a main road with a width of more than $30 \mathrm{~m}$, and the distance from the hospital façade to the road in each case is less than $6 \mathrm{~m}$. The main entrances open into the lobby, all of which have outpatient registration and dispensary counters. Some of the hospitals surveyed have a drug or convenience store adjacent to the lobby. The sizes of the lobbies and the nature of the adjacent spaces in the hospitals surveyed are listed in Table 1. 


\subsection{Questionnaire survey of noisiness}

Based on the information above, information regarding several subjective responses was requested in the survey. First, do patients or their companions located in the lobby perceive the place to be noisy? And if so, are the noise sources grouped by a factor analysis according to the hospital's routine activities? Also, is it possible for the surveyed lobby visitors to rank the perceived noisiness of one or even all of these activities? A five-point Likert scale was offered in the questionnaire to assess the level of noisiness (PARASURAMAN et al., 1991).

The responses were collected from patients and their companions (i.e. any above 13 years of age) who were willing to participate in the survey. The subjects were asked to evaluate twelve audible events inside the hospital (Table 2). The questions were posed in the form of "Do you agree that [each noise event] in this hospital is a serious problem?" Responses were made according to a five-point scale, with the specific response options being: 1) "Disagree strongly", 2) "Disagree somewhat", 3) "Neutral", 4) "Agree somewhat", and 5) "Agree strongly". The entire questionnaire, including personal information (age, sex, and the reason for visiting the hospital), took less than 5 minutes for each subject to complete. The total number of subjects was 584 . For each hospital, at least 28 people participated in the survey (Table 1). The subjective responses obtained by this questionnaire survey were classified for noisiness according to definitions established by BERGLUND et al. (1975). The arithmetic means of noisiness were calculated using the subjective responses from the subjects in each hospital's lobby and averaging the five-point scales for the 12 noise events. Figure 2 shows the noisiness results with the standard deviation (SD, noted by an error bar). The SD values were calculated for the individual responses at each of the 16 hospitals; noisiness variance differed between hospitals, even in those with an equal noisiness rating (e.g. hospital J and $\mathrm{K}$ ). The results of analysis of variance (ANOVA) tests showed significant differences in mean noisiness between individual subjects across hospitals $\left(F_{1,15,0.05}=3.72, p<0.001\right)$. The ANOVA results also showed significant differences between the mean noisiness of the 12 noise events across hospitals

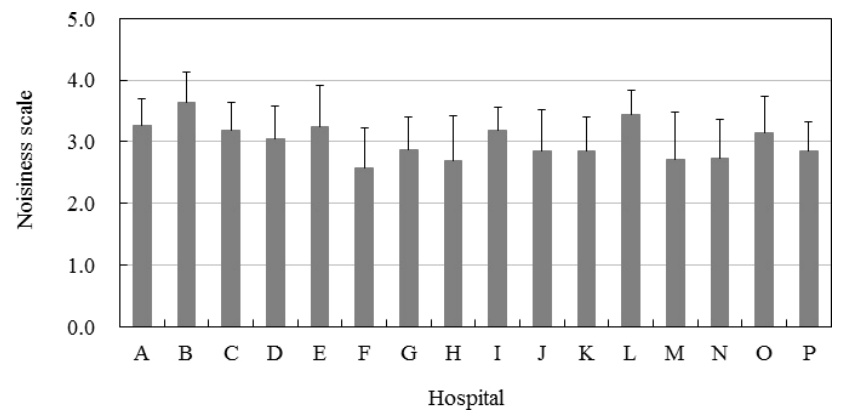

Fig. 2. The noisiness with their standard deviation of each hospital according to the questionnaire responses of patients and those accompanying them measured at that lobbies.

Table 2. Factor analysis results of noisiness and mean noisiness scales (N.S.) with standard deviation (SD) across 16 hospitals in the lobbies (Kaiser-Meyer-Olkin $(\mathrm{KMO})=0.849, n=584$ ).

\begin{tabular}{|c|c|c|c|c|c|}
\hline Item & $\begin{array}{l}\text { Factor } \\
\text { loading }\end{array}$ & Eigenvalue & $\begin{array}{c}\text { Percent } \\
\text { of total } \\
\text { variance } \\
\text { explained }\end{array}$ & N.S. & $\begin{array}{c}\text { SD of N.S. } \\
\text { among } \\
\text { hospitals }\end{array}$ \\
\hline \multicolumn{6}{|l|}{$\begin{array}{l}\text { Factor 1: Primary noise } \\
(N=6)(\text { Cronbach's } \alpha=0.834)\end{array}$} \\
\hline Facilities (elevator, aeration fan etc.) & 0.769 & \multirow{6}{*}{4.64} & \multirow{6}{*}{$38.6 \%$} & 2.60 & 0.33 \\
\hline $\begin{array}{l}\text { Broadcasting } \\
\text { (commercial or occasional channels) }\end{array}$ & 0.761 & & & 2.87 & 0.25 \\
\hline Medical appliances & 0.740 & & & 2.54 & 0.29 \\
\hline Roll call (electronic or oral) & 0.696 & & & 2.91 & 0.26 \\
\hline Entrance (door, transport) & 0.639 & & & 2.78 & 0.27 \\
\hline Ambulance (emergency service) & 0.497 & & & 2.85 & 0.29 \\
\hline \multicolumn{6}{|l|}{$\begin{array}{l}\text { Factor 2: Accidental noise } \\
(N=5)(\text { Cronbach's } \alpha=0.746)\end{array}$} \\
\hline Construction activity & 0.779 & \multirow{5}{*}{1.37} & \multirow{5}{*}{$11.5 \%$} & 3.14 & 0.31 \\
\hline Children playing & 0.774 & & & 3.17 & 0.22 \\
\hline Traffic & 0.668 & & & 2.92 & 0.23 \\
\hline Conversation (counter) & 0.572 & & & 3.15 & 0.18 \\
\hline TV & 0.524 & & & 2.63 & 0.24 \\
\hline Footfall (item dropped) & 0.307 & - & - & 2.84 & 0.28 \\
\hline
\end{tabular}


$\left(F_{1,15,0.05}=4.85, p<0.001\right)$. The mean noisiness of the 12 noise events associates well with factor loadings in the factor analysis results (Table 2). These results indicate that the subjective noisiness scale use in this study for the 12 noise events between 16 hospitals was statistically reliable in expressing the experienced annoyance rating.

Table 2 displays the result of the factor analysis for each individual audible event and the mean noisiness and SD values between hospitals. Factor 1 , which can be interpreted as "primary noise", shows high loading for events and equipment such as noise induced by medical appliances (e.g. carts, sickbeds, medical record cabinets, pulse or fever examination equipment etc.), broadcasting (e.g. departmental announcements) and facilities (air conditioners, elevators, escalators, TVs etc.). These were expressed as regular and continual noise events in the hospital lobbies. Factor 2 shows high loading for children playing and construction noise and can be regarded as "accidental noise". Such accidental noise events can unexpectedly interrupt a conversation or train of thought and are a common occurrence in urban Taiwanese hospitals. The results of the factor analysis showed that $50.1 \%$ of the variance in the noisiness can be explained by the two factors above, with the combined eigenvalues for the two factors being greater than 1.0 and each of the factor loadings being greater than 0.50 . Reliability analysis (Cronbach's alpha) was conducted to test the reliability and internal consistency of each factor.

\subsection{Noise measurements}

To measure the noise levels in the hospital lobbies, A-weighted equivalent sound pressure levels $\left(L_{A e q}, T\right)$ using time averages at 15 -min intervals were obtained over the daytime period of $9 \mathrm{am}-5 \mathrm{pm}$ because the $L_{A e q, 15 \mathrm{~m}}$ levels were required to have a normal distribution in order to explain the significant findings without accidental sound events. As indicated in Fig. 3,

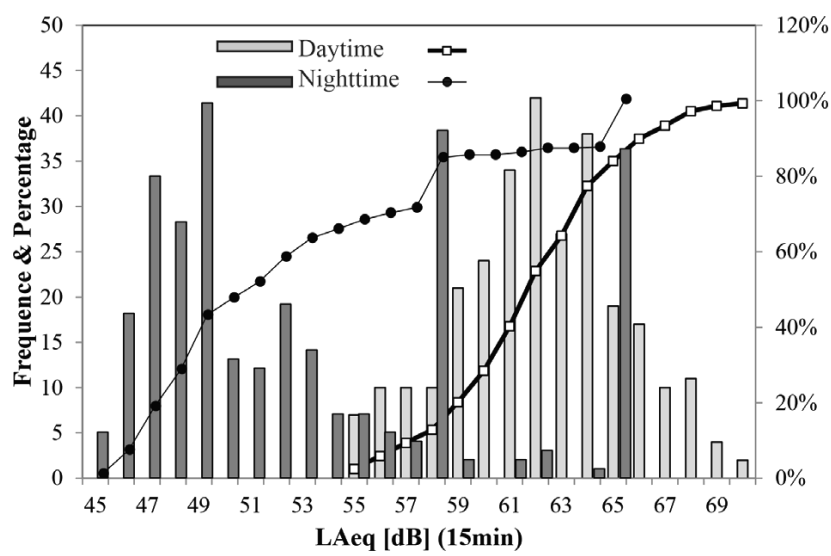

Fig. 3. A comparative cumulative frequency of the levels of $L_{A e q}$ at each 15-min interval over the daytime $(9-17)$ and the nighttime (17-24) in the 16 surveyed hospital lobbies. only the measures for the daytime period comprised something close to a normal distribution, whereas the levels measured for the nighttime period were not stationary, which would not allow to calculate the regression formulae. These results were different from the measured results for ICUs reported by RYHERD et al. (2008), in which both the daytime and nighttime measures were stationary owing to the intensive care activities occurring on a 24-hours basis. In addition, it was found that the mean interval of the restorative period $\left(L_{\text {Aeq, } 1 \mathrm{~m}}\right.$, below $\left.50 \mathrm{~dB}\right)$ was more than 9 and $13 \mathrm{~min}$ for daytime and nighttime, respectively. This explains why noise events were averaged at 15-min intervals in this study to detect the noise events without an impact wave. The measurements, taken only on rain-free weekdays, were made with a fast time constant $(0.125 \mathrm{~s})$. The octave-band levels for each hospital lobby were concurrently obtained. As shown in Fig. 4, two microphones (Brüel and Kjær, B\&K type 4190) were used to record noises at one of two receiver positions. The first position was located in the center of the given lobby's visitor seating area at least $1 \mathrm{~m}$ away from the walls and at a height of $1.2 \mathrm{~m}$, with the goal of recording all the sounds that would be experienced by a visitor surrounded by the lobby's soundscape. The other microphone was located outside of the lobby's main entrance and played the role of a road-traffic noise detector. During all the measurements, the patients, the staff members, and the patients' companions continued with their normal activities. Data were collected with B\&K PULSE system and corresponding analysis was conducted using B\&K 2-CHs. CPB analyzer software.

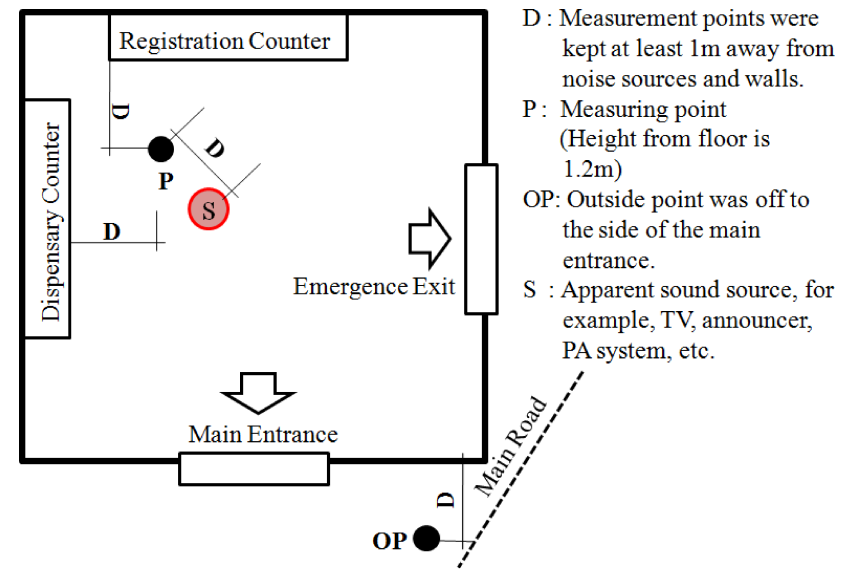

Fig. 4. Measurement positions inside and outside of a hospital lobby.

Furthermore, the reverberation time was determined based on the first $30 \mathrm{~dB}$ decay of a broadband noise (T30) generated by a B\&K Pulse system using a unidirectional loudspeaker (Norsonic - Nor276 with Nor280 amplifier) located in the lobby at least $1 \mathrm{~m}$ away from the walls but not center position. Data acquisition was performed using an FFT analyzer (B\&K, 
PULSE system) with a free field input module (B\&K, type 4190). A further calculation of the sharpness and unbiased annoyance (UBA) (FASTL, ZwICKER, 1999) was derived from the broadcasting scenarios in duration of each $30 \mathrm{~s}$ at the same position as $L_{\text {Aeq }}$ measurements over 9-12 in 16 lobbies, which is a combination metrics of sharpness $(\mathrm{S})$, fluctuation strength $(\mathrm{F})$, $N_{10}$ level, and includes a correction factor for time of day/night factor. The hospital lobby UBA survey was specifically arranged due to the results of the psychological questionnaire.

\subsection{Repetitive feature of noise levels}

To evaluate the disturbances caused by the various hospital noises, the normalized ACF (NACF) effective duration of noise levels $\left(L_{A e q}, 15 \mathrm{~m}\right)$, denoted by $\tau_{e}$ values $(h)$, was calculated to detect noise variation in this study. The autocorrelation function (ACF) is defined by

$$
\Phi_{p}(\tau)=\frac{1}{2 T} \int_{-T}^{+T} p^{\prime}(t) p^{\prime}(t+\tau) \mathrm{d} t
$$

where $p^{\prime}(t)=p(t) s(t)$, in which $p(t)$ is the sound pressure and $s(t)$ is the ear sensitivity. For convenience, $s(t)$ may be chosen as the impulse response of the $A$-weighted network. The value $\tau$ represents the time delay $(h)$, and the value $2 T$ is the integration interval. The normalized autocorrelation function (NACF) is expressed by

$$
\phi(\tau)=\Phi_{p}(\tau) / \Phi_{p}(0),
$$

where $\Phi(0)$ represents the ACF at delay time $\tau=0$ as the maximum $\Phi(\tau)$. The $\tau_{e}$ values were defined by the ten-percentile delay (at $-10 \mathrm{~dB}$ ) obtained practically from the decay rate extrapolated in the range from $0 \mathrm{~dB}$ to $-10 \mathrm{~dB}$ of the logarithmic NACF modulus. Namely, the $\tau_{e}$ values $(h)$ were calculated with a sampling rate of 0.25 hours (Fig. 1) against alpha rhythm of the brainwave signals, $0.01 \mathrm{~s}$. It has previously been shown that the $\tau_{e}$ of brain waves ( $\alpha$-wave) correlates well to the subjective preference of musical sound fields (Ando, Kageyama, 1977), since the longer $\tau_{e}$ of $\mathrm{NACF}$ in the $\alpha$-wave frequency range has been found to correspond to the fine structure of persistently occuring brain waves ( $\alpha$-wave) (ANDO, CHEN, 1996).

\section{Results}

\subsection{Evaluation of the physical parameters of noise}

\section{A. Equivalent sound pressure levels}

Figure 6 illustrates the time-dependence of the $L_{\text {Aeq, } 15 \mathrm{~m} \text { measurements for each of the } 16 \text { hospital }}$ lobbies over $9-5$ (daytime). The figure not only graphs the sound levels themselves but also the variations in sound levels, which differ greatly. In general, the sound levels in the morning were relatively high compared to those in the afternoon. Several hospitals experienced quieter periods from around 12-2 (daytime) due to fewer activities occurring during the lunchtime period. However, the road-traffic noise levels from 12-2 were just as loud, on average, as they were for the entire 9-5 period. The average levels at the 15-min intervals for the 16 hospitals ranged from $62-71 \mathrm{~dB}$, averages which were higher than the 53-58 dB averages measured at 1-min intervals in ICUs by RYHERD et al. (2008), at a position near the patients. The insulation loss (IL) value for each building in which the 16 hospital lobbies were located was obtained based on the indoor position ( $\mathrm{P}$ positions in Fig. 4) and outdoor position (OP in Fig. 4) measurements of $L_{A e q, 15 \mathrm{~m}}$ for each hospital lobby, which are illustrated in Fig. 5. The correla-

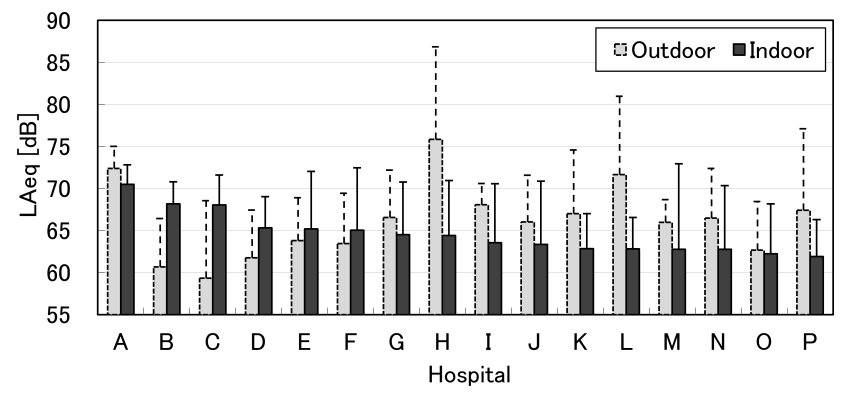

Fig. 5. Logarithmic average $L_{A e q}$ values for the 16 hospital lobbies (for indoor and outdoor measurements). The vertical bars indicate the respective daily ranges.

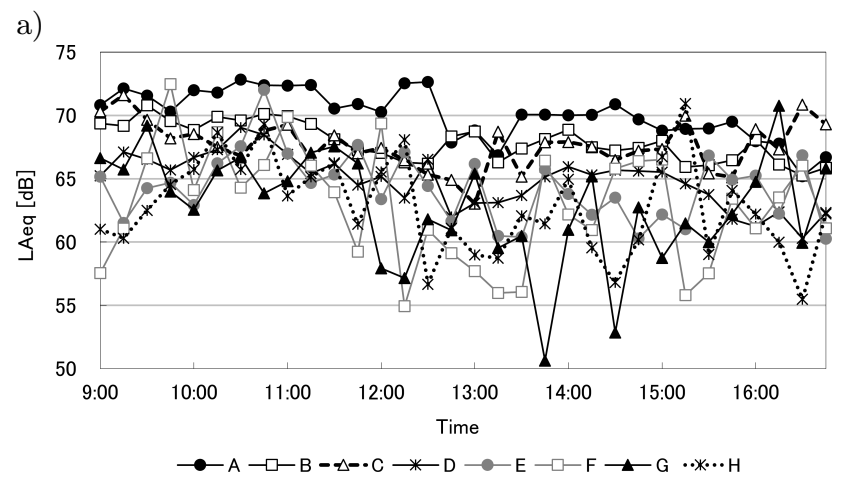

b)

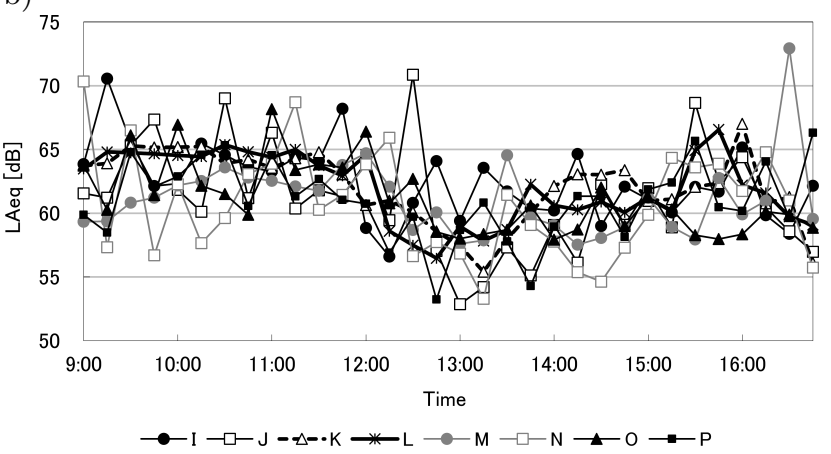

Fig. 6. Time variations in $L_{A e q}$ values for the 16 hospital lobbies (a: A - H; b: I - P). As the plots overlap considerably, these have been divided into two graphs. 
tion coefficient between the IL value and the level of psychological irritation was only -0.37 , and the mean values of the indoor and outdoor test points were still only 0.43 .

By comparing Figs. 2 and 6, it can be seen that not only were the hospital lobbies with higher $L_{A e q}$ values rated as also having higher noisiness, they were also the lobbies with narrower ranges of $L_{A e q, 15 \mathrm{~m}}$. For instance, the maximum $L_{A e q, 15 \mathrm{~m}}$ values of all measures fell within a range of $66-73 \mathrm{~dB}$ and the minimum values fell within a range of $51-65 \mathrm{~dB}$, while a wider range 45-90 dB was measured in ICUs (RYHERD et al., 2008 ) It was thus believed that the $L_{A e q, 15 \mathrm{~m}}$ values of all the measures would not serve as good indicator of psychological noisiness in this study. As indicated in Table $3, L_{A e q, 15 \mathrm{~m}}$ was correlated with noisiness, but other measures were more closely linked to noisiness, as explained by the correlation coefficient $(r)$ and the statistical reliability between the noisiness scale and the average $L_{A e q, 15 \mathrm{~m}}$ values measured inside of lobbies, which was $0.46(p=0.07)$.

On the other hand, no other factor correlated to psychological noisiness could be detected from Table 1. For example, the highest averaged noise levels were measured at hospital A, but the lobby in that hospital has fewer connections with other functional spaces besides outpatient departments. However, the architecture conditions of the hospital lobbies show that multiplications of each lobby's area, ceiling height, number of entrances and number of inpatient beds correlated roughly with the psychological noisiness $(r=0.64, p<0.01)$. We supposed, then,

Table 3. Correlation coefficient $(r)$ with $p$ value results between noise criteria and subjective noisiness in lobbies.

\begin{tabular}{|c|c|c|c|c|c|}
\hline \multicolumn{2}{|r|}{ Item } & \multicolumn{2}{|c|}{ Correlation $(r)$} & \multicolumn{2}{|c|}{ Fit $\left(r^{2}\right)$} \\
\hline \multicolumn{6}{|l|}{ Noise level $[\mathrm{dBA}]$} \\
\hline \multicolumn{2}{|c|}{ Average $L_{A e q}, 15 \mathrm{~m}$} & \multicolumn{2}{|l|}{0.46} & \multicolumn{2}{|c|}{0.22} \\
\hline \multicolumn{2}{|c|}{$L_{A e q, 15 \mathrm{~m}}$ standard deviation $\sigma_{L A e q}$} & \multicolumn{2}{|c|}{$-0.76^{* * *}$} & \multicolumn{2}{|c|}{0.58} \\
\hline \multicolumn{2}{|l|}{$L_{\max }$} & \multicolumn{2}{|l|}{-0.13} & \multicolumn{2}{|c|}{0.02} \\
\hline \multicolumn{2}{|l|}{$L_{\min }$} & \multicolumn{2}{|c|}{$0.69^{* *}$} & \multicolumn{2}{|c|}{0.48} \\
\hline \multicolumn{2}{|c|}{ Insulation of building against traffic noise } & \multicolumn{2}{|l|}{-0.37} & & \\
\hline \multicolumn{6}{|c|}{ Autocorrelation function $(\mathrm{ACF})[\mathrm{h}]$} \\
\hline \multicolumn{2}{|c|}{$\tau_{e}$ of $\mathrm{NACF}$ in $L_{A e q, 15 \mathrm{~m}}$} & \multicolumn{2}{|c|}{$0.76^{* *}$} & \multicolumn{2}{|c|}{0.58} \\
\hline \multicolumn{6}{|c|}{ Percentile $L_{\text {Aeq }}$ values $\left(L_{N}\right)[\mathrm{dBA}]$} \\
\hline \multicolumn{2}{|l|}{$L_{5}$} & \multicolumn{2}{|l|}{0.33} & \multicolumn{2}{|c|}{0.11} \\
\hline \multicolumn{2}{|l|}{$L_{10}$} & \multicolumn{2}{|l|}{0.56} & \multicolumn{2}{|c|}{0.31} \\
\hline \multicolumn{2}{|l|}{$L_{50}$} & \multicolumn{2}{|c|}{$0.69^{*}$} & & \\
\hline$L_{90}$ & & 0.71 & & & \\
\hline Compounded indi & & & & & \\
\hline$L_{\min }-L_{\max }$ & $\tau_{e} /\left(L_{\max }-L_{\min }\right)[\mathrm{h} / \mathrm{dB}]$ & $0.79^{* * *}$ & $0.86^{* * *}$ & 0.62 & 0.75 \\
\hline$L_{95}-L_{5}$ & $\overline{\tau_{e} /\left(L_{5}-L_{95}\right)}$ & $0.74^{* *}$ & $0.89^{* * *}$ & 0.55 & 0.77 \\
\hline$L_{90}-L_{10}$ & $\tau_{e} /\left(L_{10}-L_{90}\right)$ & $0.73^{* *}$ & $0.87^{* * *}$ & 0.53 & 0.75 \\
\hline & $\tau_{e} / \sigma_{L A e q}$ & & $0.85^{* * *}$ & & 0.72 \\
\hline & $N /\left(L_{5}-L_{95}\right)[$ sone $/ \mathrm{dB}]$ & & $0.85^{* * *}$ & & 0.72 \\
\hline Noise criteria & & & & & \\
\hline $\mathrm{NC}$ (Noise $\mathrm{cr}$ & on) & 0.41 & & & \\
\hline $\mathrm{RC}$ (room cri & & 0.45 & & & \\
\hline Average loud & $N$ [sone $]$ & 0.64 & & & \\
\hline Percentile lou & ss, $N_{5}$ [sone] & 0.73 & ** & & \\
\hline & $N_{10}[$ sone $]$ & 0.72 & ** & & \\
\hline Sharpness (A & $30 \mathrm{~s}$ & 0.45 & & & \\
\hline Unbiased anr & nce $(\mathrm{UBA})_{30 \mathrm{~s}}$ & 0.71 & ** & & \\
\hline T30 $(500 \mathrm{~Hz})$ & & 0.56 & * & & \\
\hline Multiplicatio & rchitecture conditions) & 0.64 & ** & & \\
\hline
\end{tabular}

Notes: ${ }^{* * *} p<0.001,{ }^{* *} p<0.01,{ }^{*} p<0.05$. 
that the architecture conditions of the hospital lobbies would serve as a potential index of psychological noisiness with regard to sound intelligibility. As sound intelligibility was assumed to most affect noisiness, and intelligibility is in turn influenced by T30, the T30 values for each lobby were thus measured according to WHO guideline (BERGLUND et al., 1999, page 56).

\section{B. Statistical levels}

It is necessary to move onto measures which relate to the variation of the sound pressure levels. The calculated results of the $L_{A e q}$ percentile values, denoted by $L_{N}$, are equivalent to the sound levels that exceeded N\% of the $L_{90}$, and $L_{\text {min }}$ denoted the minimum $L_{\text {Aeq, } 15 \mathrm{~m}}$ values of all the measures in each hospital, with the correlation coefficients $(r)$ being 0.71 $(p<0.01)$ and $0.69(p<0.01)$, respectively. The $L_{90}$ levels of all the measures fell in a narrow range from 56-68 dB, while the measure in ICUs (RYHERD et al., 2008) was $55 \mathrm{~dB}$ with a mean interval of $1 \mathrm{~min}$. As shown in Table $3, L_{90}$ was well correlated with noisiness independently, so it was assumed that $L_{\min }$ and $L_{90}$ should be similar. The trend of the correlation coefficients in Table 3 obviously increases as the $\mathrm{N} \%$ values of the $L_{N}$ levels rise. The $L_{A e q}$ values denoted in Fig. 6 nonetheless show numerous "accidental noises" during the time period from $9-5$. We confirmed through a correlation of $L_{\max }$, however, that these accidental noises did not represent primary noise impacts in the subjective evaluations discussed in Subsec. 2.2. Finally, the variation of $L_{A e q, 15 \mathrm{~m}}$ for all the measures throughout the daytime period (9-5) was found to be more important than the averaged $L_{A e q, 15 \mathrm{~m}}$ in the case of time-varying complicated noise environments.

As shown in Table $3, L_{\min }-L_{\max }, L_{95}-L_{5}$, $L_{90}-L_{10}$, and the effective duration of the normalized autocorrelation function $\left(\tau_{e}, \mathrm{NACF}\right)$ of all the measurements made at 15 -min intervals were calculated and used as indices expressing the variation of the noise levels. The correlation coefficients $(r)$ between the noisiness scale and the $L_{\min }-L_{\max }, L_{95}-L_{5}$, $L_{90}-L_{10}$, and $\tau e$ values were $0.79(p<0.001), 0.74$ $(p<0.01), 0.73(p<0.01)$ and $0.76(p<0.01)$, respectively. As shown in Fig. 7 , the $L_{\min }-L_{\max }$ values serve as a fine indicator for the variations in noisiness over time in the 16 hospital lobbies. Table 3 shows the relationships between the noisiness scale and the objective noise criteria stated above, and reveals the ranking of the connections between them.

\section{Frequency distribution}

Figure 8 shows the logarithmic average $L_{e q}, 15 \mathrm{~m}$ for each of the 16 hospital lobbies in each octave band throughout the daytime period (9-5). With the

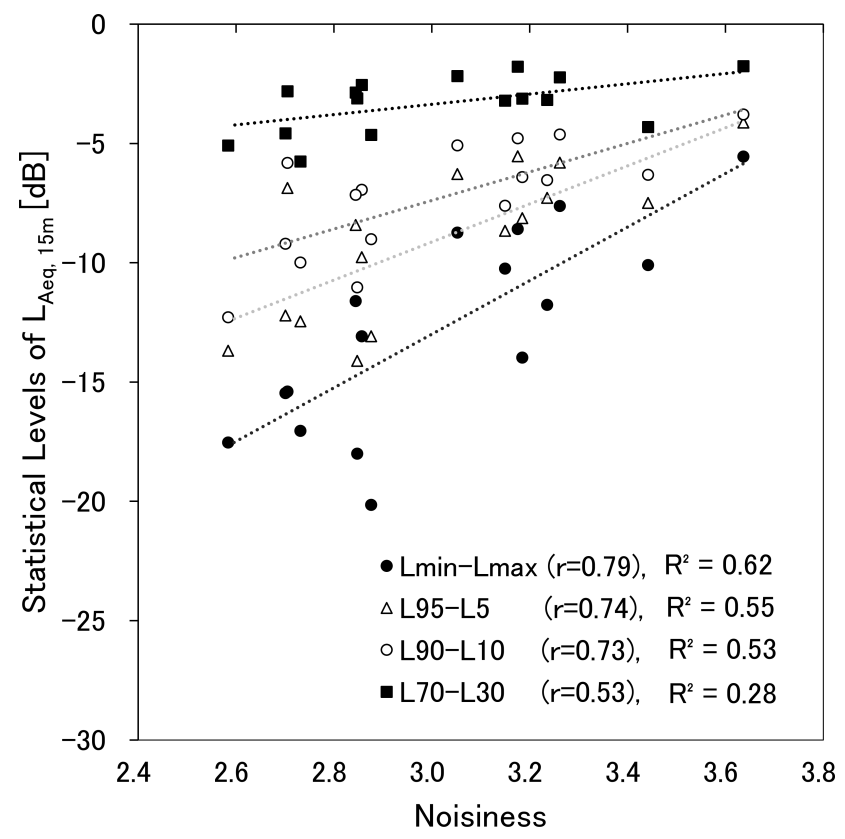

Fig. 7. Relationships between the noisiness values obtained from questionnaire responses and the $L_{\min }-L_{\max }, L_{95}-L_{5}$, $L_{90}-L_{10}$ and $L_{70}-L_{30}$ values.

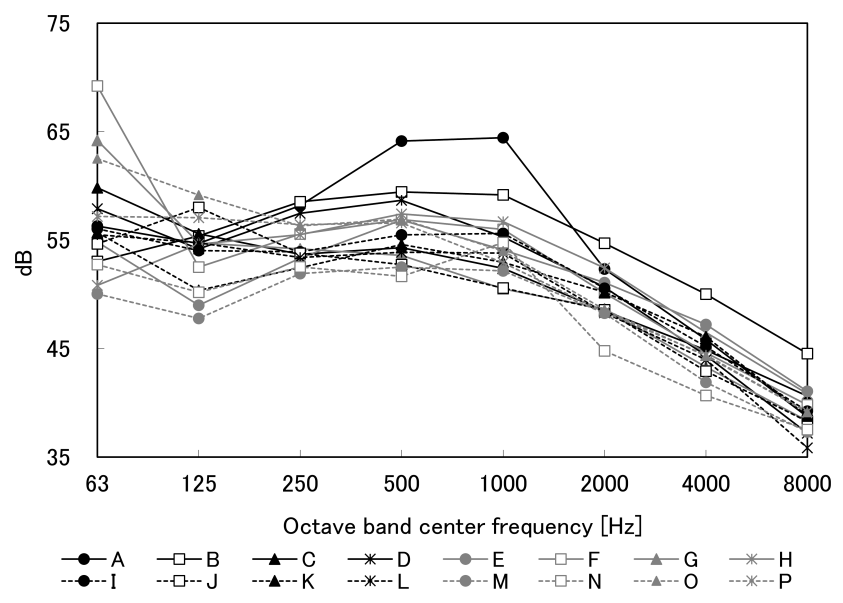

Fig. 8. Octave band spectra of noise levels measured in each hospital lobby.

exception of the highest curve, measured at hospitals A and B, the noise spectra were very similar in shape. The correlation coefficient $(r)$ between the noisiness scale obtained in the questionnaires and each $\mathrm{NC}$ (noise criterion) value (ANSI, 2008) was $0.46(p=$ 0.12 ), while the correlation coefficient $(r)$ between the noisiness scale obtained in the questionnaires and the $\mathrm{RC}$ (room criteria) values (BERANEK, 1989) was 0.45 $(p=0.08)$. These spectra profiles are similar to those measured at the Pediatric Intensive Care Unit (PICU) in Johns Hopkins hospitals before reconstruction by Busch-Vishniac et al. (2005). As they pointed out, the spectra were generally flat over $63-2000 \mathrm{~Hz}$ octave bands, with higher sound levels at lower frequencies, and with a gradual roll-off above $2000 \mathrm{~Hz}$ in some hospitals. The average noise levels of flat spectra re- 
gion were both equal to $55 \mathrm{~dB}$, even though this region became narrower over 125-1000 Hz octave bands in this study. And it was confirmed that the magnet facilities were not the primary noise source in the lobbies. These flat sound spectra regions referred to above generally encompass the speech band. Then, speech intelligibility finally becomes a potential descriptor of subjective noisiness in a hospital, and the surveys of the T30 values for each lobby were indeed required.

\section{Zwicker's noise evaluations}

The daily variation of loudness differs from $L_{A e q}$. As indicated in Table 3, the average loudness, $N$, correlated better $(r=0.64, p<0.01)$ with the noisiness than the average of $L_{A e q}(r=0.46, p=0.07)$ and the percentile loudness of $N_{5}$ correlated better to the noisiness $(r=0.73, p<0.01)$. Inversely, KACZMAREK and PREIS (2010) found that according to their annoyance assessments for simulated road traffic noise scenarios, $N$ better correlated to the annoyance ratings than the percentile loudness of $N_{5}$ or $N_{10}$. As indicated in Table 3, the unbiased annoyance (UBA) variable better correlates $(r=0.71, p<0.01)$ with the psychological noisiness, even it was not better than the percentile loudness, $N_{5}$, and $N_{10}$ values. The UBA values were derived only from the broadcasting scenarios averaged by $30 \mathrm{~s}$ in 16 hospital lobbies, and so they are not truly representative of the complex noises within the 9-5 period. However, the better correlations reveal that broadcasting constituted a potentially hazardous noise for speech interference in the hospital lobbies, in much the same way that bus noises, among various environmental noises, were found to cause annoyance in a study of environmental city noise by ABO-QUDAIS, ABU-QDAis (2005).

\section{E. Reverberation times}

Concerning the reverberant effects on conversation at hospital lobbies, the remaining analysis focuses on the reverberation time (T30) at each lobby (BUSCHVISHNIAC et al., 2005); the relevant data was measured with an FFT analyzer (B\&K, PULSE system). The results of the measurements showed that the T30 at $0.5 \mathrm{kHz}$ and $1.0 \mathrm{kHz}$ ranged from $0.5 \mathrm{~s}$ to $2.3 \mathrm{~s}$. These values encompass the speech band, obviously. At hospital F only, the T30 value was $1.0 \mathrm{~s}$ at $1.25 \mathrm{kHz}$, which was the highest level of all as we found the noisiness level to be the lowest at hospital F, as shown in Fig. 2. Figure 9 shows T30 in octave bands. The noisiness ratings of hospital lobbies with reverberation times of less than $1.5 \mathrm{~s}$ were lower than those with reverberation times greater than $1.5 \mathrm{~s}$, specifically in the cases of hospitals F, G, M, and N. Thus, as shown in Table 3 , the noisiness ratings obtained from the questionnaire responses correlated roughly with T30 when aver-

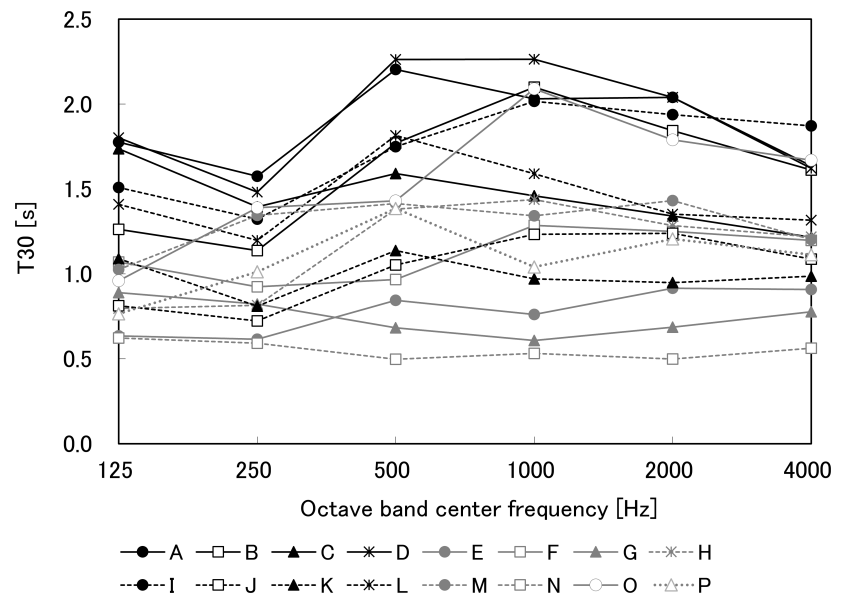

Fig. 9. Reverberation time (T30) of the 16 hospital lobbies.

aged for octave bands at $0.5 \mathrm{kHz}$ and $1 \mathrm{kHz}(r=0.56$, $p<0.05)$.

The measurement results showed that the $L_{A e q, 15 \mathrm{~m}}$ values ranged from 51-73 dBA. Furthermore, all the measured average sound levels (62-71 dBA) exceeded typical speech in conversation, which ranges from $45-$ $50 \mathrm{dBA}$. This implies that the staff needed to raise their voices to be heard above the noise. BRADLEY et al. (1999), mentioned that ambient noise levels greater than $60 \mathrm{dBA}$, corresponding to a signal with a noise ratio $\mathrm{S} / \mathrm{N}$ of less than $-5 \mathrm{~dB}$, would correspond to levels that would make conversation nearly impossible. They also pointed out that a reverberation time greater than $2.0 \mathrm{~s}$ would not represent conditions likely to occur in spaces where speech is likely. Speech audibility from the public address system and in conversations is a prerequisite for lobby settings. Thus, higher reverberation levels lead to higher conversation levels; as such, a lower reverberation time would be a necessity for better speech intelligibility. MACLEOD et al. (2007), demonstrated the effect of installing sound-absorbing panels for the noisy environments of hospital wards. Their study showed that sound pressure levels after installation had decreased by $5 \mathrm{~dB}$. The immediate impact of these sound-absorbing panels was to permit a hospital population to engage in quieter conversations while still being well understood.

\subsection{The sensation of similar prolonged noise}

As summarized in Table 3, the effective duration of normalized autocorrelation function $\left(\tau_{e}, h\right)$ of noise levels over the period of 9-5 with an integration interval of 8 hours, correlated well with the noisiness scales generated by the questionnaire. The correlation coefficient was $0.76(p<0.05)$, which was lower than that calculated for $L_{\min }-L_{\max }$. However, we found that weighting each statistical index using $\tau_{e}$ values, the psychological noisiness correlation coefficients for these compounded factors became enhanced. As shown 
in Table 3, the correlation coefficients between the $\tau_{e}$ values divided by $L_{\max }-L_{\min }, L_{5}-L_{95}, L_{1}-L_{9}$ and $L_{3}-L_{7}$ and subjective noisiness were obviously enhanced to $0.86(p<0.001), 0.89(p<0.001), 0.87$ $(p<0.001)$ and $0.77(p<0.01)$. Clearly, these 3 independent variables, $\tau_{e}$ values, $1 /\left(L_{5}-L_{95}\right)$ and percentile loudness, $N_{5}$, were the best choices to be investigated by the multiple linear regression with noisiness scales. The regression model of the noisiness scale (N.S.) in a given hospital lobby over the period of $9-5$ could be calculated as:

$$
\begin{aligned}
\text { N.S. }= & 2.71 /\left(L_{5}-L_{95}\right) 15 \mathrm{~m}+0.78 \tau_{e, 15 \mathrm{~m}} \\
& +0.03 N_{5,15 \mathrm{~m}}+1.90,
\end{aligned}
$$

where 1.90 denotes the intercept with regard to the scale range of noisiness in present study. The multiple correlation coefficient $r=0.91\left(F_{3,12,0.05}=19.8\right.$, $p<0.001)$, the fitness $R^{2}=0.83$, and the standard error estimate was 0.14 . The highlight order for this regression model (Eq. (3)) of these three variables was $1 /\left(L_{5}-L_{95}\right), \tau_{e}$, and $N_{5}$, since the factor loadings were $0.43,0.38$ and 0.28 respectively. Forward stepwise regression was used to screen the independent variables listed in Table 3; the results indicated that the ideal regression model should include variable T30, the correlation was enhanced to $r=0.92\left(F_{4,11,0.05}=16.7\right.$, $p<0.001$ ), and the fitness was enhanced to $R^{2}=0.86$. However, T30 was not corrected for noise measures and only practically applied in the case of indoor environments. For backward stepwise regression model, the variable of $N_{5}$ would be eliminated and the correlation was decreased to $r=0.86\left(F_{2,13,0.05}=23.5\right.$, $p<0.001)$ and the fitness was decreased to $R^{2}=0.78$.

As the multiple linear regression suggests, the statistical levels, $1 /\left(L_{5}-L_{95}\right)_{15 \mathrm{~m}}, \tau_{e} 15 \mathrm{~m}$ values, and percentile loudness, $N_{5,15 \mathrm{~m}}$ of noise events were the primary descriptors of subjective noisiness for the complexity of noise sources over a long period (9-5) in hospital lobbies which is different from the sensation of an instant annoyance.

\section{Conclusions}

1. The noisiness ratings obtained from the questionnaire cannot be explained only by the average $\mathrm{L}_{A e q}$. As shown in Table 3, the analysis showed that the noisiness ratings were well correlated with the $L_{\text {min }}$ and the 90 percentile $L_{A e q}$ values. In particular, the predicted N.S. value was highly correlated with the multiple linear regression model $(r=0.91$, $\left.R^{2}=0.83\right)$ formed by 3 orthogonal variables: statistical levels $1 /\left(L_{5}-L_{95}\right)_{15 \mathrm{~m}}$, the effective duration of the normalized autocorrelation function $\left(\tau_{e}, h\right)$, and percentile loudness, $\mathrm{N}_{5}$ of all $L_{A e q, 15 \mathrm{~m}}$ measurements.

2. Previous studies found that the highest annoyance ratings were obtained for even traffic distribution in a laboratory setting, and the most clustered distribution resulted in the lowest annoyance rating. Similarly, in this study, $\tau_{e}$ values $(h)$ calculated according to the range of noise levels indicated by $L_{5}-L_{95}$ showed the highest correlation with noisiness $\left(r=0.89, R^{2}=0.77\right)$. This means that hospital lobbies with fewer quiet periods and that exhibited continuously similar noise features could be rated as noisier.

3. We collected samples of the ceiling material for all the hospital lobbies surveyed and found that the absorptive ceiling tiles used in hospital $\mathrm{F}$ caused the noisiness ratings for that lobby to be clearly lower than the ratings for the other lobbies, as shown in Fig. 2. Preventing high reverberation with absorptive materials is the best solution to the excessive noise levels in hospital lobbies, since the use of automatic doors leading from a lobby to another space is illegal in Taiwan due to emergency-related considerations

4. The unbiased annoyance $\mathrm{UBA}_{30 \mathrm{~s}}$ derived from the broadcasting scenario in the lobbies with $30 \mathrm{~s}$ intervals can serve as an optimal physical parameter for measuring specific temporary noise sources $\mathrm{UBA}_{30} \mathrm{~s}$ was not the best descriptor for psychological noisiness over daytime hours $(r=0.71, p<0.01)$.

\section{Acknowledgments}

I would like to express my thanks to the many people who have, in various ways, helped me in the course of preparing this study. In particular, I wish to thank Professor Shinichi Sato, who kindly gave me directions on my references and data analysis. I am also indebted to the Architecture and Building Research Institute, Ministry of the Interior, and Taiwan for their two year period of financial support to complete this research. Special thanks are due to my many colleagues for their participation in the experiments involving the subjective judgments and the noise recordings.

\section{References}

1. Abo-Qudais S., Abu-Qdais H. (2005), Perceptions and attitudes of individuals exposed to traffic noise in working places, Building and Environment, 40, 6, 778787.

2. Acoustical Society of America (2008), Criteria for Evaluating Room Noise, American National Standards Institute (ANSI), S12, 2.

3. Ando Y., Chen C. (1996), On the analysis of autocorrelation function of $\alpha$-waves on the left and right cerebral hemispheres in relation to the delay Time of single sound reflection, Journal of Architectural Planning and Environmental Engineering, Architectural Institute of Japan (AIJ), 488, 67-73. 
4. Ando Y., Kageyama K. (1977), Subjective preference of sound with a single early reflection, Acustica, 37, 111-117.

5. BERANEK L.L. (1956), Criteria for office quieting based on questionnaire rating studies, Journal of the Acoustical Society of America, 28, 833-852.

6. BERANEK L.L. (1989), Balanced noise criterion curves, $N C B$, Journal of the Acoustical Society of America, 86, 650-664.

7. Berglund B., Berglund U., Lindvall T. (1975) Scaling loudness, noisiness, and annoyance of aircraft noise, Journal of the Acoustical Society of America, $\mathbf{5 7}, 930-934$.

8. Berglund B., Lindvall T., Schwela D.H. [Ed.], (1999), World Health Organization, Guidelines for community noise, p19.

9. Berglund B., Hassmen P., Preis A. (2002), Annoyance and spectral contrast are cues for similarity and preference of sounds, Journal of Sound and Vibration, 250, 53-64.

10. Busch-Vishniac I.J., West J.E., Barnhill C., Hunter T., Orellana D., Chivukula R. (2005), Noise levels in Johns Hopkins Hospital, Journal of the Acoustical Society of America, 118, 3629-3645.

11. Bradley J.S., Reich R.D., Norcross S.G. (1999) On the combined effects of signal-to-noise ratio and room acoustics on speech intelligibility, Journal of the Acoustical Society of America, 106, 1820-1828.

12. De Coensel B., De Muer T., Yperman I., BotTELDOOREN D. (2005), The influence of traffic flow $d y$ namics on urban soundscapes, Applied Acoustics, 66, 175-194.

13. Griffiths I.D., Langdon F.J. (1968), Subjective response to road traffic noise, Journal of Sound and Vibration, 8, 1, 16-33.

14. International Organization for Standardization ISO 532 (1975), Acoustics - Method for Calculating Loudness Level.

15. Kaczmarek T., Preis A. (2010), Annoyance of timevarying road-traffic noise, Archives of Acoustics, 35, 3, 383-393.

16. Kracht J.M., Busch-Vishniac I.J., West J.E. (2007), Noise in the operating rooms of Johns Hopkins Hospital, Journal of the Acoustical Society of America, 121, 2673-2680.

17. Macleod M., Dunn J., Busch-Vishniac I.J., West J.E., ReEdy A. (2007), Quieting Weinberg 5C: A case study in hospital noise control, Journal of the Acoustical Society of America, 121, 3501-3508.
18. McJury M.J. (1995), Acoustic noise levels generated during high field MR imaging, Clinical Radiology, 50, 331-334.

19. Orellana D., Busch-Vishniac I.J., West J.E. (2007), Noise in the adult emergency department of Johns Hopkins Hospital, Journal of the Acoustical Society of America, 121, 1996-1999.

20. Parasuraman A.A., Zethaml V.A., Berry L.L. (1991), Refinement and reassessment of the SERVQUAL Scale, Journal of Retailing, 67, 420-405.

21. Ryherd E.E., WAye K.P., LuUngkvist L. (2008), Characterizing noise and perceived work environment in a neurological intensive care unit, Journal of the Acoustical Society of America, 123, 747-756.

22. Sandrock S., Schütte M., Griefahn B. (2010), Mental strain and annoyance during cognitive performance in different traffic noise conditions, Ergonomics, 53, 8, 962-971.

23. Sato S., You J., JeOn Y. (2007), Sound quality characteristics of refrigerator noise in real living environments with relation to psycho- acoustical and autocorrelation function parameters, Journal of the Acoustical Society of America, 122, 314-325.

24. TANG S.K. (1997), Performance of noise indices in airconditioned landscaped office buildings, Journal of the Acoustical Society of America, 102, 1657-1663.

25. Topf M. (1988), Noise- induced occupational stress and health in critical care nurses, Hospital Top, 66, 30-34.

26. Torija A.J., Genaro N., Ruiz D.P., RamosRidao A., Zamorano M., Requena I. (2010), Prioritization of acoustic variables: Environmental decision support for the physical characterization of urban sound environments, Building and Environment, 45, $1477-1489$.

27. Tsiou C., Efthymiatos G., Katostaras T. (2008), Noise in the operating rooms of Greek hospitals, Journal of the Acoustical Society of America, 123, 757-765.

28. Williams A.L., Drongelen W.V., LAsky R.E. (2007), Noise in contemporary neonatal intensive care, Journal of the Acoustical Society of America, 121, 2681-2690.

29. Yamada Y., Komuro K., Nakayama S., KokuBo T., SAKurai Y. (2003), Actual conditions of noises in hospitals: On planning and conditions of wards, Journal of Institute of Noise Control Engineering of JAPAN, 27, 5, 373-382.

30. Zwicker E., FAstl H. (1999), Psychoacoustics facts and models, p. 324, Second Updated Edition, SpringerVerlag Berlin Heidelberg. 